EPiC Series in Computing
Volume 62, 2019, Pages 10-20
SuMO User Conference 2019

\title{
Modelling green waves for emergency vehicles using connected traffic data
}

\author{
Laura Bieker-Walz ${ }^{1}$ and Michael Behrisch ${ }^{1}$ \\ German Aerospace Center (DLR) \{laura.bieker, michael.behrisch\}@dlr.de
}

\begin{abstract}
For emergency vehicle drivers it is an important task to reach the incident location as fast as possible. Therefore a self-organizing green wave could help emergency vehicles to accomplish this goal. This study presents an approach how emergency vehicle can be prioritized at traffic lights and simulates the possible benefit for the emergency vehicle. Traffic data from vehicular communication can be used to find the optimal timing for the traffic light to modify the existing traffic phases and reduce the possible negative impact on other traffic participants.
\end{abstract}

\section{Introduction}

Driving an emergency vehicle is a challenging task for emergency vehicle drivers. They have to reach the incident location as fast as possible and have therefore special rights which allows them to disregard traffic rules e.g. drive fast than speed limits or violate red traffic lights.

But still emergency vehicle drivers are facing huge traffic problems. One problem is that the risk for emergency vehicles is statistically 8 times higher to have a car crash compare to other traffic participants [21]. Most of the crashes with emergency vehicles which were driving with blue flashing lights and siren occur at controlled intersection while a red traffic light phase was violated. The results of traffic safety study of 189 emergency vehicle crashes can be seen in Figure 1 and a detailed description of the traffic safety study can be found in [2].

Another problem which emergency vehicle drivers are facing is that traffic jams are blocking their routes. It is often not possible for other traffic participants to form a rescue lane to let the emergency vehicle pass the congested areas. This often causes that it is difficult to keep legal help periods.

A possible solution to support emergency vehicle drivers would be to give emergency vehicle priority at controlled intersections. This might result in faster and safer routes for emergency vehicles. In the following existing traffic management strategies for emergency vehicles are presented. Next, a new support strategy is presented and simulated with different simulation scenarios in SUMO. The paper ends with a conclusion and outlook of further work. 


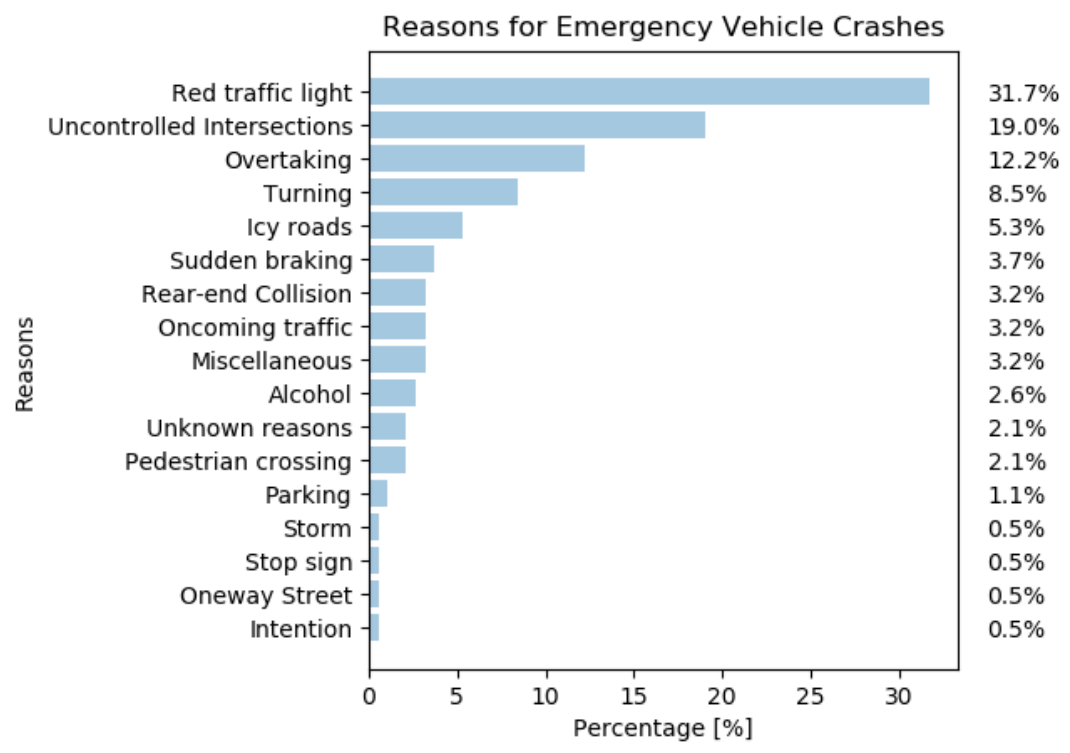

Figure 1: Reasons for crashes with emergency vehicles with blue flashing light and siren (based on the study from [2])

\section{Traffic management for emergency vehicle}

Normally, the whole traffic system should be optimized in regard of specific criteria when traffic is optimized. In contrast to this, the optimization of the routes of emergency vehicles are different because only one vehicle class should be optimized while the negative influence of the other traffic participants should be as low as possible. This section gives an overview over the existing traffic management strategies for emergency vehicles.

\subsection{Emergency vehicle warnings}

Many traffic problems like traffic accidents and blocked streets on the routes of emergency vehicles occur because other traffic participants are not realizing that an emergency vehicle is approaching even with blue flashing light and siren [13]. The easiest way to overcome this problem is to inform the traffic participants of an approaching emergency vehicle [20].

Vehicular communication can help to notice an emergency vehicle earlier for example by sending an emergency vehicle approaching warning to all traffic participants within the communication range. An advantage of this application is that it can already be used with low penetration rate of equipped vehicles. But the problem is that only equipped vehicles will receive the warning and not all traffic participants will react to the emergency vehicle.

Another way for sending warnings is via the Radio-Data-System (RDS) [4]. RDS is already used in most of the vehicles and interrupts the radio or music program to provide traffic information. Therefore the vehicles do not have to be equipped with new technologies and only vehicles in communication distance will receive the warning. But a problem is that the warning only is often not enough for the emergency vehicle to get priority. According to interviews with emergency vehicle drivers many traffic participants are panicking when they hear a siren and 
do not know where the emergency vehicle comes from and how to react appropriate [7].

\subsection{Traffic light prioritization}

In a first approach the prioritization of traffic lights for emergency vehicles was evaluated in the study of Nelson [14]. In this study the green phases of the traffic lights were manually extended to let the emergency vehicle pass the intersection. The intention of this study was to see how much the normal traffic is negatively effected by this approach. In general the negative effect can be neglected for single use (if many emergency vehicles are approaching within a short time period the travel time can increase by 20 to 30 seconds).

In a simulation study [9] a green wave for a corridor of 8 traffic lights in China was evaluated. As a result, the average travel time of the emergency vehicles could decrease by $27 \%$.

There are some countries which have already implemented prioritization systems for emergency vehicles. In Japan the system called "Fast Emergency Vehicle Preemption Systems" (FAST) is in use [12]. The emergency vehicles are detected with infrared sensors. The current position and the vehicle ID is send to traffic control center which extends green phases or shortens red phases at the traffic lights of the emergency vehicle.

In Germany there is the possibility to prioritize special traffic modes at traffic lights since 1970. Currently, the research projects HALI [16] and SIRENE [8] are addressing this topic for a test field in Berlin (HALI) and Brunswick (SIRENE). The project HALI uses the position system of GALILEO to switch the traffic lights to green for a fire brigade in Berlin. The project SIRENE uses the test field of AIM (Application Platform for Intelligent Mobility) [18] in Brunswick for the prioritization algorithm.

Another traffic management solution is the software called Sitraffic Stream from Siemens which is for example implemented in the city Böblingen (Germany) [19]. The emergency vehicles are localized by GPS. If they pass a defined virtual position, the upcoming traffic light is set to an emergency mode. During this mode the traffic light is set green to the direction of the emergency vehicle until the emergency vehicle has passed another virtual position behind the intersection.

Additionally, some fire brigades have a manual system with push buttons to request a green phase from the direction of the fire brigade at the next intersection, see an example in Figure 2. When the button is pushed the traffic light is set to green for the emergency vehicle for a fixed time (e.g. for two minutes).

In this study a new prioritization approach should be evaluated and compared to two existing approaches. Therefore the FAST and the STREAM approach have been chosen. The FAST approach was chosen because the extension of green phases is a very simple and easy to implement strategy. The STREAM approach was chosen because the general approach is similar to others and furthermore by using GPS it is not necessary to use expensive additional hardware. In the following section a new traffic management approach is presented.

\section{$3 \quad$ Emergency vehicle prioritization approach (WALABI)}

The general approach is that the emergency vehicle should receive priority at every traffic light system on its way (a so called green wave). The general approach was already presented and evaluated in [1] and [3]. To reduce the negative impact for other traffic participants the optimal distance for switching the traffic light is calculated. The following steps are performed for the emergency vehicle prioritization application: 


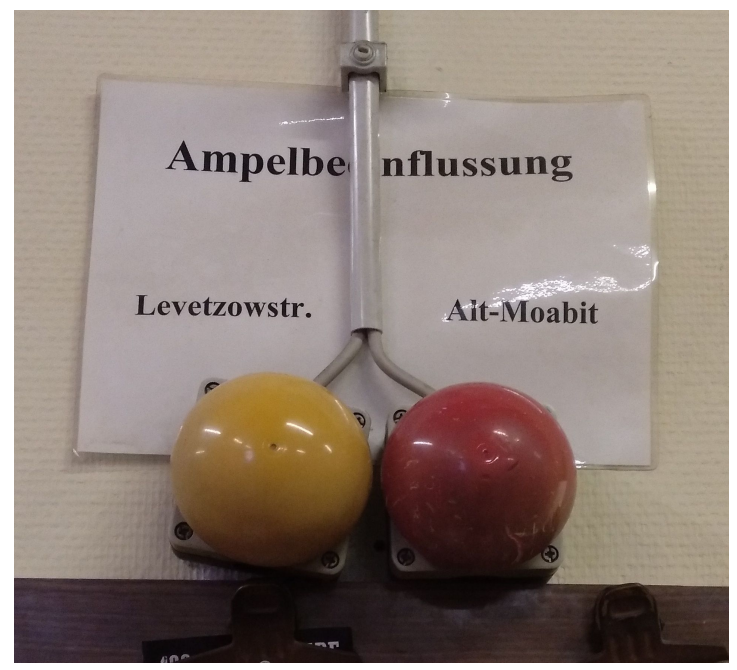

Figure 2: Example of a manual traffic management solution for emergency vehicles in a fire brigade in Berlin

- Emergency vehicle sends CAMs (Cooperative Awareness Message) and route information

- Road side unit informs Traffic management center (TMC)

- TMC sets Traffic lights on the route of the emergency vehicle

- Green for the emergency vehicle and red for all other traffic participants

- After the emergency vehicle passed the intersection normal operation continues

The general approach is called WALABI approach in the following and has two problems. First, if more than one emergency vehicle is requesting priority at a single intersection. Second, the vehicles might have to wait for a long time without knowing why the traffic light is red . If the vehicles are equipped with communication technology they will receive a message that an emergency vehicle is approaching. Otherwise they could expect after 5 minutes with a red traffic light signal that the traffic light is broken. Furthermore, a long red phase might cause traffic jams which are not easy to dissolve.

To handle the first problem that two vehicles could request priority at the same time the algorithm checks whether the routes of the emergency vehicles have same intersections. Only in this case the algorithm has to take care of conflicts. Then, the estimated time slot when the emergency vehicles will reach the conflicting intersections will be calculated. If the time slots are different the algorithm sets priority to the first emergency vehicle and afterward to the second. If both vehicles are approaching at the same time slot, the emergency vehicle with the highest priority class will get green and the other emergency vehicle will receive a warning about other emergency vehicle. The highest priority have ambulances, then fire brigades and then police cars. If also the priority of the emergency vehicles is the same. Both vehicles will receive a warning and the emergency vehicles have to pass the intersection carefully without the support of the application.

For handling the second problem the approach was extended to minimize the red traffic phase as short as possible and as long as necessary. Therefore, the optimal distance of the 


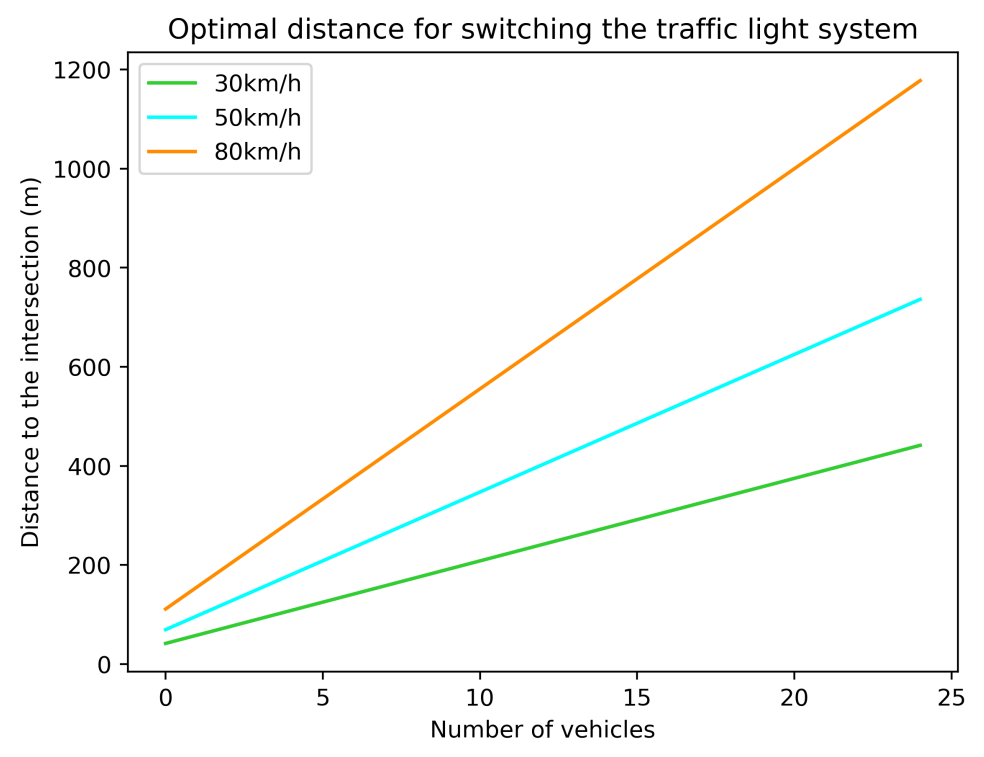

Figure 3: Optimal distance for switching the traffic light system dependent on the speed of the emergency vehicle

emergency vehicle to the intersection was calculated to switch the traffic light to green. For this the information about the vehicles which are waiting at the traffic light is necessary. This information can be used from vehicular communication, induction loops or floating car data (see [15]).

With the number of waiting vehicles at the traffic lights, the time which is needed to let them and the emergency vehicle pass the traffic light $T_{\text {free }}$ is calculated:

$$
T_{\text {free }}=(\# \text { waiting }+1) * t_{B}+t_{\text {safety }}
$$

where

\#waiting is the number of waiting vehicles

$t_{\text {safety }}$ is a safety time which is set to 3 seconds

$t_{B}$ is the required time for one vehicle to pass the intersection. If the traffic light is switched to green for more than 10 seconds and a traffic density $q_{S}$ from 2.000 vehicles per hour, the values is $t_{B}=1,8 \mathrm{~s}$ according to the German Guidelines for dimensioning road traffic facilities [6]. Other studies used for $t_{B} \approx 2 s$ [11], [17], [5] which is also used in this work.

From the time $T_{\text {free }}$ and the speed of the emergency vehicle it can be calculated the distance of the emergency vehicle to the intersection:

$$
\text { distance }=T_{\text {free }} * v_{\text {ev }}
$$

where $v_{e v}$ is the speed of the emergency vehicle $(\mathrm{m} / \mathrm{s})$

In Figure 3 is presented a diagram with the calculated distances to the traffic light dependent on the number of waiting vehicles and the speed of the emergency vehicle. Furthermore, the approach should not only handle one single intersection it should also see the whole traffic system. The approach should be able to generate a green wave for a corridor. It might happen 


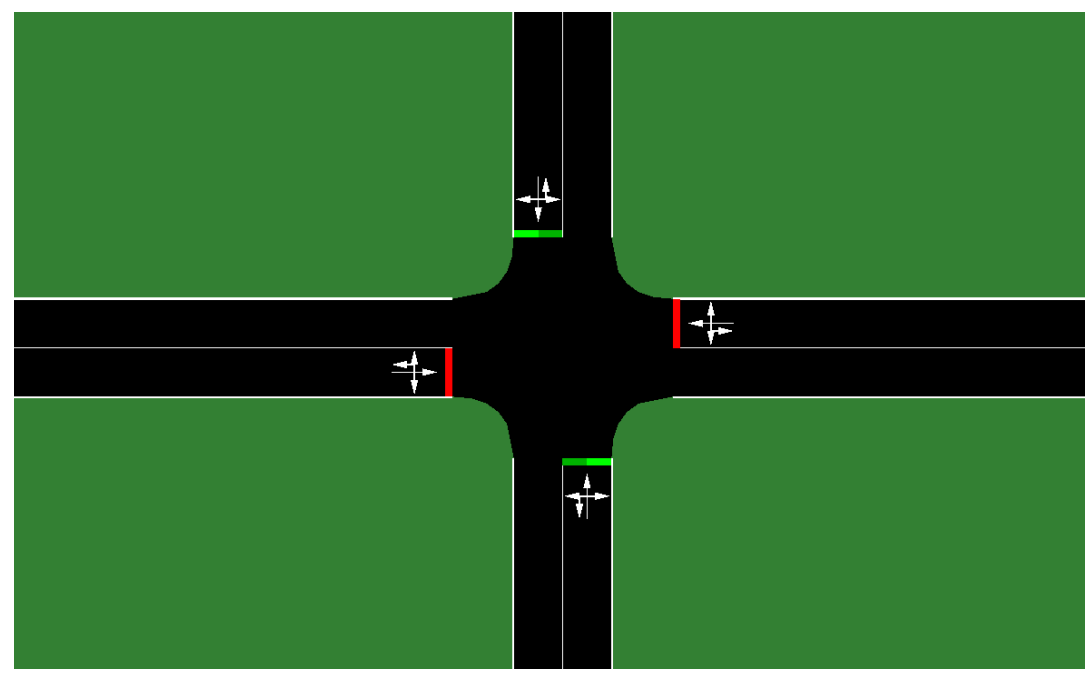

Figure 4: Simple intersection scenario in SUMO

that because of the traffic of one intersection cannot be run-off because the street after the intersection is jammed. For this reason the approach was extended. The algorithm checks also whether the next road has the capacity for the waiting vehicle:

$$
\# \text { waiting }_{1}+\# \text { waiting }_{2} \geq \text { capacity }
$$

where \#waiting ${ }_{1}$ number of waiting vehicles in front of the first intersection \#waiting 2 number of waiting vehicles of the second intersection

If this conditions applies it is necessary to set the second intersection to green more early and this formula applies:

$$
T_{\text {free } 2}=\left(\# \text { waiting }_{1}+\# \text { waiting }_{2}+1\right) * t_{B}+t_{\text {safety }}
$$

When the distance is calculated from $T_{\text {free } 2}$ it has to be added an additional time $z$ the time which is needed to pass the distance between the two intersections without waiting vehicles.

$$
\text { distance }=\left(T_{\text {free } 2}+z\right) * v_{\text {ev }}
$$

\section{Simulation results}

For this research study the traffic of a simple intersection and a corridor was simulated with SUMO version 1.0.1 [10]. In Figure 4 the simulation scenario of the simple intersection is displayed. The simple intersection has 4 roads with a length of 500 meters. The scenario was simulated with one and two lanes at each road.

The corridor scenario includes three intersection. The streets of the scenario are all 200 meters long. The routes of the emergency vehicle includes all three intersection. Every simulation scenario was simulated 100 times with different insertion times and routes of the emergency vehicle. The simulations were performed with different traffic volumes to see the boundaries of the algorithm. The travel time of the emergency vehicles will be compared to see how good the 


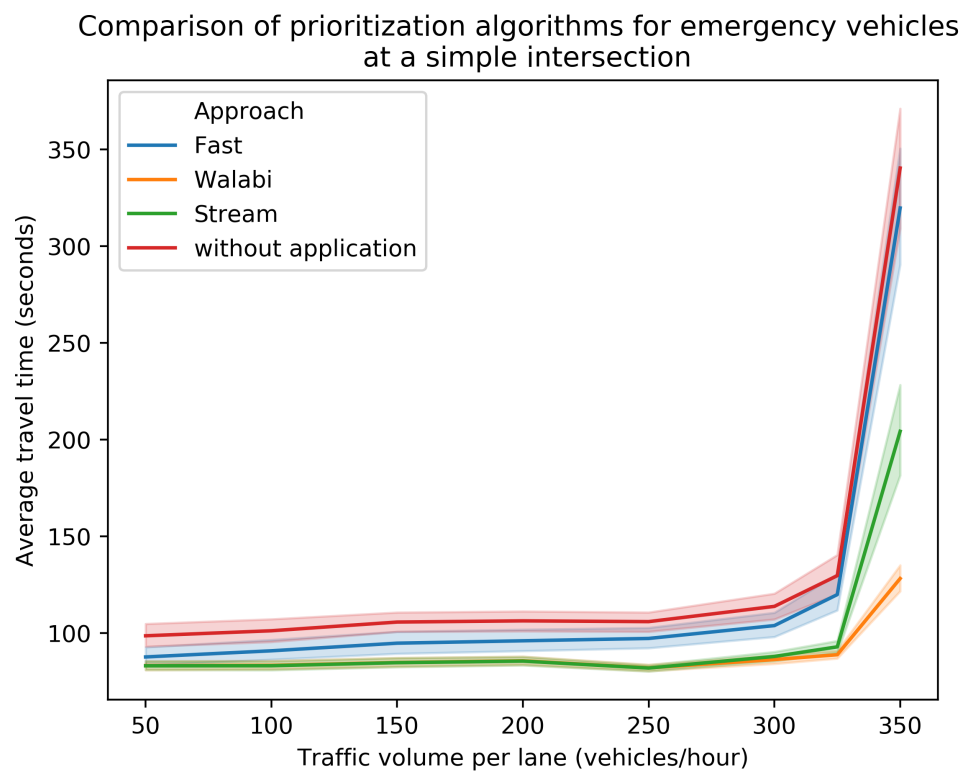

Figure 5: Simulation results of a simple intersection

algorithms are prioritizing the emergency vehicles. The best case would be that the emergency vehicle can drive the whole route with full speed and without stopping.

In Figure 5 the simulation results for a simple intersection with one lane can be seen. The results show that the FAST algorithm is always having slightly better result for the emergency vehicle compare to an emergency vehicle without application and special rights. The problem with the FAST algorithm is that it might happen that the emergency vehicle could not pass the intersection within the green time extension for example if vehicle need more time than expected. This problem was also seen in real world test fields. STREAM and WALABI have similar results regarding the average travel time. The average travel time for both algorithms are better than the results from FAST and for an emergency vehicle without application. For this simple intersection scenario the algorithm of the STREAM and the WALABI approach a very similar. The STREAM approach is always switching the traffic light at the same distance from the emergency vehicle to the traffic light. In contrast to this, the WALABI approach switchs the traffic light according to the current traffic state. If the traffic queue of the traffic light is shorter than the set distance in STREAM, then there is no difference between the the STREAM and the WALABI approach. Only for higher traffic volumes the results for the WALABI approach are showing a benefit because the algorithm is setting the traffic light to green earlier than the STREAM algorithm.

In Figure 6 the results of the simulation scenario with a simple intersection with two lanes are displayed. The variation of the average travel time of the emergency vehicles are much large in this scenario than in the simple scenario with only one lane. In the simulation with two lanes the positive effect of the WALABI approach is lower but still visible than for the scenario with only one lane because this scenario is not as crowed as the scenario with one lane.

The simulation results of the corridor scenario are shown in Figure 7. The positive benefit of the WALABI approach are much better visible here for higher traffic densities. The difference 


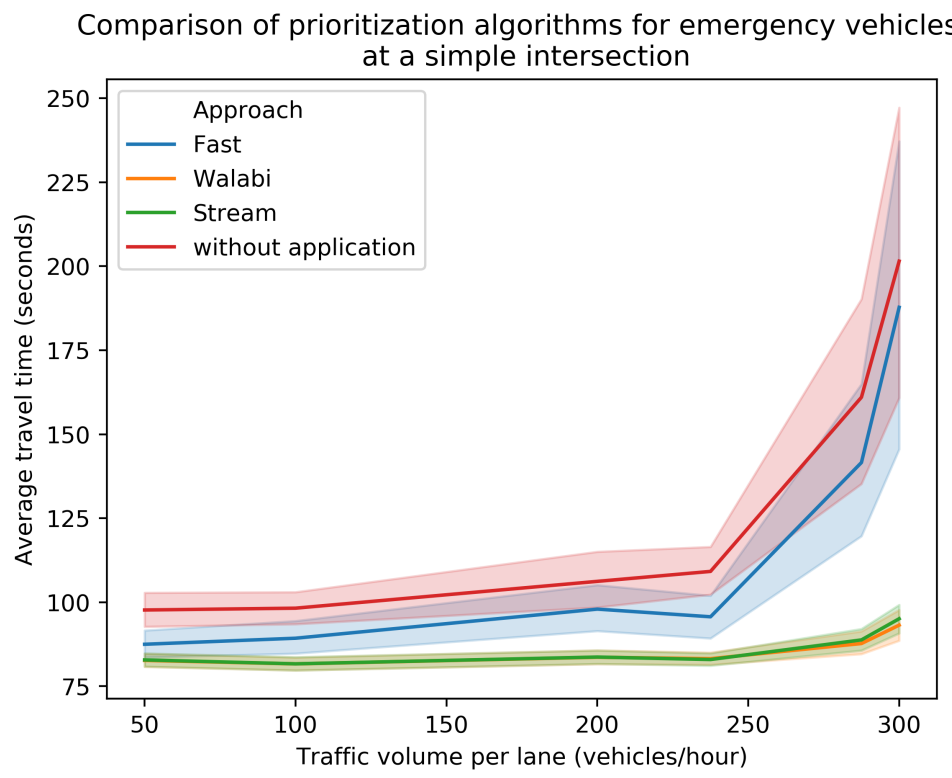

Figure 6: Simulation results of a simple intersection with 2 Lanes

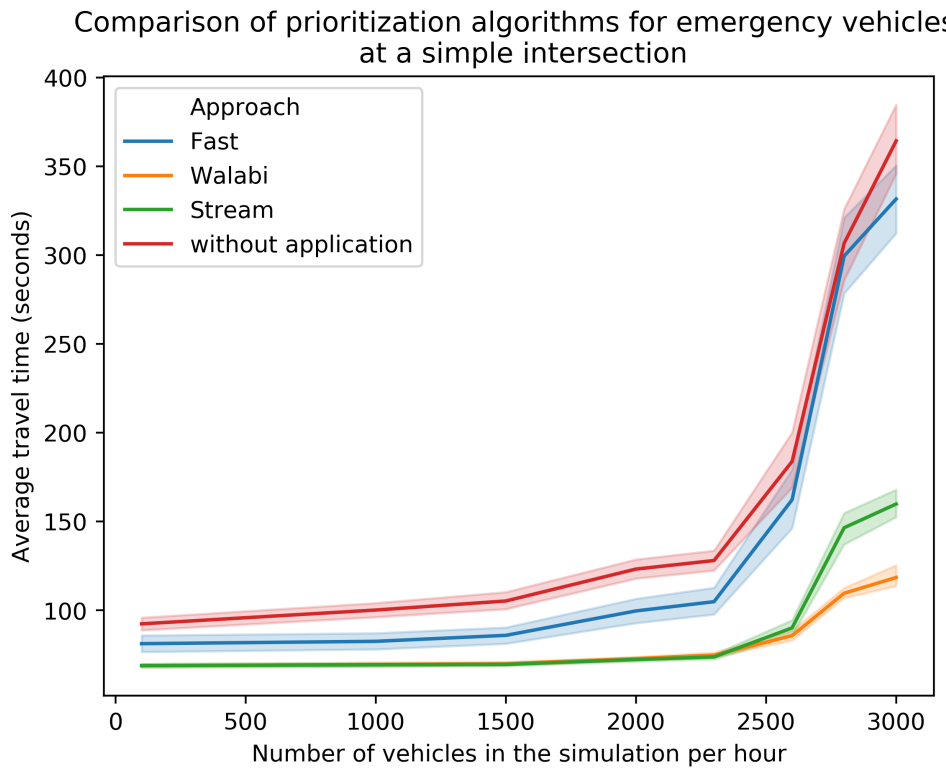

Figure 7: Simulation results of a corridor

between the STREAM and the WALABI approach is based here on the coordination of two intersections especially if the scenario is very jammed. 


\section{Conclusion and Future Work}

In this paper support strategies for emergency vehicles and a new green wave algorithm for emergency vehicles have been presented. The microscopic traffic simulation SUMO was used to compare two existing algorithm FAST and STREAM to a new algorithm called WALABI. The simulation results showed that all algorithms are having a benefit for the emergency vehicle compare to no support application. The best results regarding the average travel time of the emergency vehicles is performed by the WALABI approach. For a simple intersection the results of WALABI and STREAM are similar because the difference between both algorithms are having a bigger impact if more than one intersection is evaluated.

The results in this study also showed that at some point the traffic density is too high to prevent the emergency vehicles from stopping. In further work more simulation scenarios especially real world examples should be investigated to get a better knowledge about the limitations of this algorithm. 


\section{References}

[1] Laura Bieker. Emergency vehicle prioritization using vehicle-to-infrastructure communication. In Young Researchers Seminar, Juni 2011.

[2] Laura Bieker. Traffic safety evaluations for emgergency vehicles. In Young Researchers Seminar, Juni 2015.

[3] Laura Bieker-Walz. Cooperative traffic management for emergency vehicles in the city of bologna. In SUMO 2017 - Towards Simulation for Autonomous Mobility, volume 31 of Berichte aus dem DLR-Institut für Verkehrssystemtechnik, pages 135-141, Mai 2017.

[4] Stephan Bockting. Verkehrsunfallanalyse bei der nutzung von sonder-und wegerechten gemäß stvo. $B G W$ Forschung, 2007.

[5] Ing Manfred Boltze and Ing Axel Wolfermann. Der einfluss von zwischenzeiten auf die kapazität von lichtsignalanlagen. In Tagungsbericht zur HEUREKA. Forschungsgesellschaft für Straßen- und Verkehrswesen, März 2011.

[6] W Brilon, G Reichard, F Weisse, S Westphal, and N Wu. Handbuch für die bemessung von straßenverkehrsanlagen. Schlussbericht zum Forschungsauftrag FE, 2:R96A, 2001.

[7] Andreas Buchenscheit, Florian Schaub, Frank Kargl, and Michael Weber. A vanet-based emergency vehicle warning system. In Vehicular Networking Conference (VNC), 2009 IEEE, pages 1-8. IEEE, 2009.

[8] Institut für Automation und Kommunikation e. V. Sirene: Secure and intelligent road emergency network. https://sirene.ifak.eu/, 2018. abgerufen am 14. November 2018.

[9] Wenwen Kang, Gang Xiong, Yisheng Lv, Xisong Dong, Fenghua Zhu, and Qingjie Kong. Traffic signal coordination for emergency vehicles. In Intelligent Transportation Systems (ITSC), 2014 IEEE 17th International Conference on, pages 157-161. IEEE, 2014.

[10] Daniel Krajzewicz, Jakob Erdmann, Michael Behrisch, and Laura Bieker. Recent development and applications of sumo - simulation of urban mobility. International Journal On Advances in Systems and Measurements, 5(3\&4):128-138, Dezember 2012.

[11] Dieter Lohse and Werner Schnabel. Grundlagen der Straßenverkehrstechnik und der Verkehrsplanung: Band 2-Verkehrsplanung. Beuth Verlag, 2011.

[12] Joji Maki and Masahide Hatakeyama. Development of fast emergency vehicle preemption systems. In 18th ITS World Congress, 2011.

[13] Matthias Mändl and Jürgen Schafberger. Untersuchung der richtungsortung von akustischen warneinrichtungen für bevorrechtigte wegebenutzer im innenraum von kraftfahrzeugen, 2015. abgerufen am 1. Februar 2018.

[14] Eric Nelson and Darcy Bullock. Impact of emergency vehicle preemption on signalized corridor operation: An evaluation. Transportation Research Record: Journal of the Transportation Research Board, 1727:1-11, 2000.

[15] Thorsten Neumann. Rückstaulängenschätzung an Lichtsignalanlagen mit Floating-Car-Daten. PhD thesis, Institut für Verkehrssystemtechnik, DLR, 2011.

[16] Ronald Nippold and Robert Oertel. Hali berlin: Bevorrechtigung von sondereinsatzfahrzeugen an lichtsignalanlagen mit galileo prs, 2018.

[17] Markus Rausch. Ereignisorientierte Routenwahl in spontan gestörten Stadtstraßennetzen zur Anwendung eines selbstorganisierten Störfallmanagements. PhD thesis, Technische Universität Dresden, 2015.

[18] Lars Schnieder and Karsten Lemmer. Anwendungsplattform intelligente mobilität-eine plattform für die verkehrswissenschaftliche forschung und die entwicklung intelligenter mobilitätsdienste. Internationales Verkehrswesen, 64(4):62-63, 2012.

[19] Siemens AG. Sitraffic stream: satellitengestütztes bevorrechtigungssystem. https: //www.siemens.com/content/dam/webassetpool/mam/tag-siemens-com/smdb/mobility/ road/connected-mobility-solutions/traffic-prioritization/stream/documents/ 
sitraffic-stream-fire-brigade-v2-de-180223.pdf, 2018.

[20] Thomas Strang. Intelligent transportation systems: European c2c activities, 2010.

[21] Manfred Unterkofler and Reinhard Schmiedel. Verbesserung der sicherheit bei sondersignaleinsätzen. bergisch-gladbach, info 34/95. Wissenschaftliche Informationen der Bundesanstalt für Straßenwesen, 34/95, 1995. 